\title{
Baroreflex Function in Battery Workers Having High Blood Lead (Pb) Level
}

\author{
Pradeep Kumar* ${ }^{1}$, Shyam Vinay Sharma ${ }^{2}$, Sciddhartha Koonwar ${ }^{3}$, and \\ Virendra Atam ${ }^{4}$ \\ 1. Associate Professor, Department of Physiology, $K G$ 's Medical University Lucknow India \\ 2. Research Scholar, Department of Medicine, $K G$ 's Medical University Lucknow India \\ 3. Assistant Professor, Department of Pediatrics, $K G$ 's Medical University Lucknow India \\ 4. Professor, Department of Medicine, KG's Medical University Lucknow India
}

\section{Introduction}

The cardiovascular system has the crucial task of supplying all the body cells with their metabolic needs. In human control mechanism is well developed to maintain the blood pressure and flux within adequate levels. The arterial baroreflex regulate the blood pressure and maintain circulation to the brain and other organs 1. Baroreceptors sense systemic blood pressure indirectly, by the extent of stretch of receptors in the walls of the carotid arteries and of the aorta. Increase in blood pressure elicits reflex parasympathetic activation and sympathetic inhibition, with subsequent decreases in heart rate (HR), cardiac contractility, vascular resistance, and venous return. Conversely, a decrease in arterial pressure reduces baroreceptor afferent discharge and triggers reflex increases in HR, cardiac contractility, vascular resistance, and increased venous return. Thus the baroreflex, by affecting blood pressure and HR control, provides powerful beat-to-beat negative feedback regulation of arterial blood pressure that minimizes short-term fluctuations in pressure ${ }^{2}$.

Lead $(\mathrm{Pb})$ is one of the oldest-established poisons. However, lead exposure continues to be a major public health problem, particularly in urban area of the USA and India ${ }^{3}$. Lead's uptake by excitable cells like neurons is due in large part to its interactions with cellular mechanisms that, under ordinary conditions, perform calcium-mediated functions. Lead enters astroglia and neurons via voltage-sensitive calcium channels ${ }^{\mathbf{4}, 5}$ the predominant channel subtype depending on the specific type of cell ${ }^{6}$. Lead disrupts calcium homeostasis, causing a marked accumulation of calcium in lead-exposed cells ${ }^{78}$. Lead, in nanomolar concentrations, also induces mitochondrial release of calcium ${ }^{9}$ thus initiating apoptosis of neuronal cells. Lead accumulates in and damages mitochondria the organelles mediating cellular energy metabolism ${ }^{\mathbf{1 0}}$. Haem biosynthesis, a function of normal mitochondrial activity, is affected by lead, with disruptive effects on synaptic transmission in the brain. Decreased mitochondrial functioning also can transform ordinarily benign synaptic transmission ${ }^{11}$. Thus lead $(\mathrm{Pb})$ can modulate various reflexes of the body including baroreflex. Quantification of arterial baroreflex sensitivity (BRS) is source of valuable information for the assessment of neural cardiovascular regulation in normal and disease states. Therefore, in present study we evaluated the baroreflex sensitivity in lead exposed persons.

\section{Material and methods:}

The study was conducted on 85 subjects divided into study and control groups. The inclusion criteria for study group were: age between 20 to 40 years, exposure to Lead $(\mathrm{Pb})$ more than five years, BMI less than 25 and having no any systemic diseases. The control groups were not exposed to lead but having same age and BMI. The lead exposed subjects were taken from battery workshops of Lucknow city. Recruited subject were explained about the study protocol. Proper history was taken and clinical examination was done to include any systemic diseases.

Baseline parameters like Heart Rate (HR), Systolic and Diastolic Blood Pressure (SBP and DBP) and Electrocardiogram (ECG) were recorded. Mean Arterial pressure (MAP) and BMI were calculated. The Blood Lead Level (BLL) was estimated by Lead Care based on atomic absorption furnace in both the groups.

\section{Arterial Baroreflex Sensitivity (BRS) Assessment}

The autonomic changes may also be due to baroreflex stimulation caused by changes in arterial pressure due to the regular inspiratory increase in venous return to the heart. ${ }^{12-14}$ therefore in present study we indirectly assess the BRS by quantifying sinus arrhythmia and change in blood pressure induced by volunteer apnea and change in posture. Deep metronomic breathing at a rate of six cycles per minute is the most common and reliable test to assess respiratory sinus arrhythmia, with acceleration of heart rate during inspiration and deceleration during expiration under optimized condition. 
Slow deep breathing test: After 3-5 min in lying posture, the subjects were asked to take deep and slow breath at rate of 6 breaths per minute for 1 minute ${ }^{15}$. The duration of each respiratory cycle was ten second, with equal time for inspiration and expiration. The inspiratory and expiratory phases were indicated to the subject with green and red light indicators respectively on patient panel of CANWIN ANS System. The ECG was continuously being recorded for 60 second. The magnitude of sinus tachycardia was assesses by measuring the heart rate in inspiration.

\section{Blood pressure response to posture:}

Baseline supine blood pressure and heart rate was measured by oscilometric method. Then the subjects were asked to stand up quickly from supine posture to standing and remain standup for two minutes ${ }^{16}$ by putting equal weights on both legs and without any support. The blood pressure and heart rate was recorded at 2 minutes automatically by the machine. The difference in systolic blood pressure after 2 minutes of standing from that of lying (baseline) pressure was calculated.

\section{Observation and Results:}

One hundred thirty five subjects ( 70 control and 65 study cases) volunteered to participate in the study. The mean age of these subjects was $41.80 \pm 7.38$ and $42.07 \pm 8.30$ years in cases and controls respectively, No significance difference in body mass index in cases and control was observed. Their mean systolic and diastolic blood pressures in the sitting position prior to ECG recording were $128.63 \pm 18.26$ and81.58 $\pm 8.78 \mathrm{mmHg}$ respectively in cases showed no significant difference with controls. There was a significant increase in the HR when the posture changed from supine to standing, being highest in standing this increase was more in control $(79.43 \pm 8.78)$ than cases $(76.54 \pm 10.78 \mathrm{bpm})(P<0.05)$ (Table -II). The systolic blood pressure was recorded after two minutes of change in posture; it has been observed that change in posture, from lying to standing, causes increase in systolic blood pressure, diastolic blood pressure and mean blood pressure it showed the integrity of baroreflex. In our study the lead exposed person exhibit less increase in above parameters. Another parameter for assessment of BRS was induction of sinus arrhythmia by deep breathing test; our data showed that increase in heart rate in lead exposed persons was significantly lower than controls $(\mathrm{p}<0.05)$.

Baseline Parameters of the Cases and Controls

\begin{tabular}{|c|c|c|c|}
\hline Parameters & Cases $(\mathbf{n}=\mathbf{6 5})$ & Controls $(\mathbf{n}=\mathbf{7 0})$ & P - value \\
\hline Age (yr) & $41.80 \pm 7.38$ & $42.07 \pm 8.30$ & $P>0.05$ \\
\hline BMI (kg/m2) & $25.41 \pm 4.70$ & $26.99 \pm 3.65$ & $P>0.05$ \\
\hline SBP (mm Hg) & $128.63 \pm 18.26$ & $124.14 \pm 5.10$ & $P>0.05$ \\
\hline DBP $(\mathbf{m m}$ Hg) & $81.58 \pm 8.78$ & $79.52 \pm 4.81$ & $P>0.05$ \\
\hline HR (Beat/ min) & $71.23 \pm 11.15$ & $72.7 \pm 3.46$ & $P<0.05$ \\
\hline BLL (mg/dl) & $35.45 \pm 13.36$ & $6.04 \pm 1.49$ & $* P<0.0001$ \\
\hline MAP & $95.80 \pm 8.73$ & $96.43 \pm 10.33$ & $P>0.05$ \\
\hline
\end{tabular}

Table-II

MAP and HR in response to change in posture

\begin{tabular}{|c|c|c|c|}
\hline Parameters & Cases $(\mathbf{n}=\mathbf{6 5})$ & Controls $(\mathbf{n}=\mathbf{7 0})$ & P - value \\
\hline HR & $76.54 \pm 10.78$ & $79.43 \pm 8.78$ & $P>0.05$ \\
\hline SBP & $125.75 \pm 10.39$ & $129.72 \pm 5.81$ & $P<0.05$ \\
\hline DBP & $84.56 \pm 4.70$ & $86.34 \pm 3.48$ & $P>0.05$ \\
\hline MAP & $95.25 \pm 4.41$ & $101.23 \pm 4.98$ & $P<0.05$ \\
\hline
\end{tabular}

Table -III

Effect of Lead on sinus tachycardia

\begin{tabular}{|c|c|c|c|}
\hline Parameters & Cases $(\mathbf{n}=\mathbf{6 5})$ & Controls $(\mathbf{n}=\mathbf{7 0})$ & P - value \\
\hline Baseline HR & $70.23 \pm 11.15$ & $72.7 \pm 3.46$ & $\mathrm{P}>0.05$ \\
\hline HR in Inspiration & $75.58 \pm 9.34$ & $81.79 \pm 8.42$ & $\mathrm{P}<0.05$ \\
\hline
\end{tabular}




\section{Discussion:}

In this study, we sought to determined weather Lead $(\mathrm{Pb})$ overload caused by Lead $(\mathrm{Pb})$ exposure affect baroreflex function in north Indian Battery Workers. Our data showed that the blood lead level (BLL) was significantly higher $(\mathrm{P} \leq 0.001)$ in battery workers. The BMI and Age was similar in case (study group) and controls. Baseline data showed that there was more significant difference in HR, SBP, DBP, and MAP.

The baroreflex sensitivity was assessed by induction of blood Pressure change by change in posture and initiation of sinus arrhythmia Arterial baroreceptor play a crucial role in adjustment of the cardiovascular system to several surrounding condition ${ }^{1}$. Baroreceptor stimulation result in increase /decrease in blood pressure and heart rate, which is mediated by sympathetic and parasympathetic activity, Hence the baroreflex reactivity. Quantification of arterial baroreflex sensitivity is sources of information about cardiovascular adjustment. Chronic lead overload leads to dysfunction of cardiovascular system ${ }^{17}$. It had also observed that lead exposure causes decrease in nerve conduction velocity ${ }^{18}$. In an experimental study on rat showed that lead intoxication causes significant changes in morphological disturbance in myelin sheath ${ }^{19}$. In our study the alteration in BRS was observed which is due to dysfunction of the baroreceptor or conduction velocity of the vagus and glassopharyngial nerve or due to change in medullary nucleus. This preliminary study did not able to explain the cause in decrease in baroreflex but probably due to decrease in conduction velocity of sympathetic and parasympathetic nerve because lead exposure may cause oxidative stress and disruption of morphology of nerve ${ }^{20}$.

\section{Conclusion:}

Our study data suggests that Lead $(\mathrm{Pb})$ exposure causes decrease in baroreflex sensitivity in battery workers which may leads to orthostatic hypotension and deregulation of cardiovascular hemodynamic

\section{Acknowledgement:}

The authors are grateful to the heads of the battery Industries / workshops and the other subjects in the workshops who participated in the study. Dr. R.C. Murthy, Scientist and HOD, Analytical Chemistry Section, I.I.T.R. Lucknow, U.P., India to highly appreciate.

\section{References:}

[1]. Sleight P, Eckberg DL.1992 Human Baroreflexes in Health and Disease. (Clarendon, Oxford)

[2]. Pang CCY (2001) Autonomic control of the venous system in health and disease. Effects of drugs. Pharmacol Ther 90:179-230.

[3]. Tong S, von Schirnding YE, Prapamontol T. Environmental lead exposure: a public health problem of global dimensions. Bull World Health Organ 2000; 78: 1068-77.

[4]. Kerper LE, Hinkle PM. Lead uptake in brain capillary endothelial cells: activation by calcium store depletion. Toxicol Appl Pharmacol 1997a; 146:127-33.

[5]. Legare ME, Barhoumi R, Hebert E, Bratton GR, Burghardt RC, Tiffany-Castiglioni E. Analysis of Pb2+ entry into cultured astroglia. Toxicol Sci 1998; 46: 90-100.

[6]. Audesirk G. Electrophysiology of lead intoxication: effects on voltage sensitive ion channels. Neurotoxicology 1993;14:137-47.

[7]. Bressler JP, Goldstein GW. Mechanisms of lead neurotoxicity. Biochem Pharmacol 1991; 41: 479-84

[8]. Bressler J, Kim KA, Chakraborti T, Goldstein G. Molecular mechanisms of lead neurotoxicity. Neurochem Res 1999; 24: 595-600.

[9]. Silbergeld EK. Mechanisms of lead neurotoxicity, or looking beyond the lamppost. FASEB J 1992; 6: 3201 -6.

[10]. Anderson AC, Pueschel SM, Linakis JG. Pathophysiology of lead poisoning. In: Pueschel SM, Linakis JG, Anderson AC, editors. Lead poisoning in children. Baltimore (MD): P.H. Brookes; 1996. p. 75-96.

[11]. Beal MF, Brouillet E, Jenkins BG, Ferrante RJ, Kowall NW, Miller JM, et al. Neurochemical and histologic characterization of striatal excitotoxic lesions produced by the mitochondrial toxin 3- nitropropionic acid. J Neurosci 1993; 13: 4181-92.

[12]. Mrowka R, Persson PV, Theres H, Patzak A, Blunted arterial baroreflex causes "pathological" Heart rate turbulence, Am J Physiol Regul Integr Comp Physiol, 2000; 279: 1171-5.

[13]. Segerson NM, Wasmund SL, Abedin M, et al., Heart rate turbulence parameters correlate with post -PVC changes in muscle sympathetic nerve activity, Heart Rhythm, 2007;4: 284-9.

[14]. Bauer A, Malik M, Schmidt G, etal. Heart rate turbulence: standard of measurements. Physiological interpretation, and Clinical use: International Society for Holter and Non invasive Electrophysiology Consensus, J Am Coll Cardiol, 2008; 52: 1353-65.

[15]. Sundkwist G, ALMER LO, Lilja B. Respiratory influence on heart rate in diabetes mellitus. Br. Med.J.1979; 1,924-25.

[16]. Smit AA,Wieling W, Karemaker JM. Clinical Approach to cardiovascular reflex testing. Clin Sci. (Lond) .1996; 91Suppl:108-12.

[17]. Chand Basha D, Sadak Basha S, Rajarami Reddy G, Lead-induced cardiac and hematological alterations in aging Wistar male rats: alleviating effects of nutrient metal mixture August 2012, Volume 13, ISSUE 4, pp 359-368.

[18]. Singer R, Valciukas JA, Lilis R. Lead exposure and nerve conduction velocity: the differential time course of sensory and motor nerve effects. NEUROTOXICOLOGY. 1983 Summer;4(2):193-202.

[19]. DA Purser, K R Berrill, and S K Majeed. Effects of lead exposure on peripheral nerve in the cynomolgus monkey. Br J Ind Med. 1983 November; 40(4): 402-412.

[20]. B. Dabrowska-bouta, G. Sulkowski, G. Bartosz, M. Walski, U. Rafalowska Chronic lead intoxication affects the myelin membrane status in the central nervous system of adult rats. Journal of Molecular Neuroscience. 1999, Volume 13, ISSUE 1-2, pp 127-139. 